\title{
Erratum to: The phenotypic spectrum of organic acidurias and urea cycle disorders. Part 2: the evolving clinical phenotype
}

Stefan Kölker ${ }^{1}$ - Vassili Valayannopoulos ${ }^{2}$ • Alberto B. Burlina ${ }^{3}$ • Jolanta Sykut-Cegielska ${ }^{4}$ - Frits A. Wijburg ${ }^{5}$. Elisa Leão Teles ${ }^{6} \cdot$ Jiri Zeman $^{7} \cdot$ Carlo Dionisi-Vici $^{8} \cdot$ Ivo Barić $^{9} \cdot$ Daniela Karall $^{10}$ • Jean-Baptiste Arnoux ${ }^{2}$. Paula Avram $^{12}$ - Matthias R. Baumgartner ${ }^{13}$ - Javier Blasco-Alonso ${ }^{14}$. S. P. Nikolas Boy ${ }^{1} \cdot$

Marlene Bøgehus Rasmussen ${ }^{11}$ • Peter Burgard ${ }^{1} \cdot$ Brigitte Chabrol $^{15} \cdot$ Anupam Chakrapani $^{16} \cdot$ Kimberly Chapman $^{17}$.

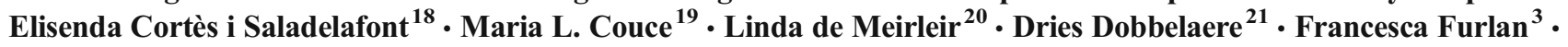
Florian Gleich $^{1}$ - Maria Julieta González ${ }^{18}$ - Wanda Gradowska ${ }^{22} \cdot$ Stephanie Grünewald $^{23}$ - Tomas Honzik $^{7}$. Friederike Hörster $^{1}$ - Hariklea Ioannou ${ }^{24}$ - Anil Jalan ${ }^{25}$ • Johannes Häberle ${ }^{13}$ • Gisela Haege ${ }^{1}$. Eveline Langereis ${ }^{5}$. Pascale de Lonlay $^{2} \cdot$ Diego Martinelli $^{8} \cdot$ Shirou Matsumoto $^{26} \cdot$ Chris Mühlhausen $^{27} \cdot$ Elaine Murphy $^{28} \cdot$ Hélène Ogier de $^{2}$ Baulny $^{29} \cdot$ Carlos Ortez $^{18}$ - Consuelo C. Pedrón ${ }^{30}$ • Guillem Pintos-Morell ${ }^{31} \cdot$ Luis Pena-Quintana $^{32}$. Danijela Petković Ramadža ${ }^{32}$ • Esmeralda Rodrigues ${ }^{6}$ - Sabine Scholl-Bürgi ${ }^{10} \cdot$ Etienne Sokal $^{34}$. Marshall L. Summar ${ }^{17} \cdot$ Nicholas Thompson $^{23} \cdot$ Roshni Vara $^{35} \cdot$ Inmaculada Vives Pinera $^{36} \cdot$ John H. Walter $^{37}$. Monique Williams $^{38}$ • Allan M. Lund ${ }^{11}$ - Angeles Garcia Cazorla ${ }^{18}$

Published online: 16 June 2015

(C) SSIEM 2015

Erratum to: J Inherit Metab Dis DOI 10.1007/s10545-015-9840-x

The name of Angeles Garcia Cazorla is not correctly stated. It must read Angeles Garcia-Cazorla, and A. Garcia-Cazorla

The online version of the original article can be found at http://dx.doi.org/ 10.1007/s10545-015-9840-x.

Stefan Kölker

Stefan_Koelker@med.uni-heidelberg.de

1 Department of General Pediatrics, Division of Inherited Metabolic Diseases, University Children's Hospital Heidelberg, Im Neuenheimer Feld 430, 69120 Heidelberg, Germany

2 Hôpital Necker-Enfants Malades, Assistance Publique-Hôpitaux de Paris, Reference Center for Inherited Metabolic Disease, Necker-Enfants Malades University Hospital and IMAGINE Institute, Paris, France 
3 Azienda Ospedaliera di Padova, U.O.C. Malattie Metaboliche Ereditarie, Padova, Italy

4 Screening Department, Institute of Mother and Child, Warsaw, Poland

5 Department of Pediatrics, Academisch Medisch Centrum, Amsterdam, Netherlands

6 Unidade de Doenças Metabólicas, Serviço de Pediatria, Hospital de S. João, EPE, Porto, Portugal

7 First Faculty of Medicine Charles University and General University of Prague, Prague, Czech Republic

8 Ospedale Pediatrico Bambino Gésu, U.O.C. Patologia Metabolica, Rome, Italy

9 School of Medicine University Hospital Center Zagreb and University of Zagreb, Zagreb, Croatia

10 Medical University of Innsbruck, Clinic for Pediatrics I, Inherited Metabolic Disorders, Innsbruck, Austria

11 Centre for Inherited Metabolic Diseases, Department of Clinical Genetics, Copenhagen University Hospital, Rigshospitalet, Copenhagen, Denmark

12 Institute of Mother and Child Care "Alfred Rusescu", Bucharest, Romania

13 Division of Metabolism and Children's Research Centre, University Children's Hospital Zurich, Steinwiesstraße 75, 8032 Zurich, Switzerland

14 Hospital Materno-Infantil (HRU Carlos Haya), Málaga, Spain

15 Centre de Référence des Maladies Héréditaires du Métabolisme, Service de Neurologie, Hôpital d'Enfants, CHU Timone, Marseilles, France

16 Birmingham Children's Hospital NHS Foundation Trust, Steelhouse Lane, Birmingham B4 6NH, UK

17 Children's National Medical Center, 111 Michigan Avenue, N.W., Washington, DC 20010, USA

18 Hospital San Joan de Deu, Servicio de Neurologia and CIBERER, ISCIII, Barcelona, Spain

19 Metabolic Unit, Department of Pediatrics, Hospital Clinico Universitario de Santiago de Compostela, Santiago de Compostela, Spain

20 University Hospital Vrije Universiteit Brussel, Bruxelles, Belgium
21 Centre de Référence des Maladies Héréditaires du Métabolisme de l'Enfant et de l'Adulte, Hôpital Jeanne de Flandre, Lille, France

22 Department of Laboratory Diagnostics, The Children's Memorial Health Institute, Warsaw, Poland

23 Metabolic Unit Great Ormond Street Hospital and Institute for Child Health, University College London, London, UK

24 1st Pediatric Department, Metabolic Laboratory, General Hospital of Thessaloniki 'Hippocration', Thessaloniki, Greece

25 N.I.R.M.A.N., Om Rachna Society, Vashi, Navi Mumbai, Mumbai, India

26 Department of Pediatrics, Kumamoto University Hospital, Kumamoto City, Japan

27 Universitätsklinikum Hamburg-Eppendorf, Klinik für Kinder- und Jugendmedizin, Hamburg, Germany

28 National Hospital for Neurology and Neurosurgery, Charles Dent Metabolic Unit, London, UK

29 Hôpital Robert Debré, Université de Paris, Paris, France

30 Department of Pediatrics, Metabolic Diseases Unit, Hospital Infantil Universitario Niño Jesús, Madrid, Spain

31 Department of Pediatrics, Hospital Universitari Germans Trias I Pujol, Badalona, Spain

32 University Hospital Center Zagreb, Zagreb, Croatia

33 University of Las Palmas de Gran Canaria, Hospital Universitario Materno-Infantil de Canarias, Unit of Pediatric Gastroenterology, Hepatology and Nutrition, Las Palmas de Gran Canaria, Spain

34 Cliniques Universitaires St Luc, Université Catholique de Louvain, Service Gastroentérologie and Hépatologie Pédiatrique, Bruxelles, Belgium

35 Evelina Children's Hospital, St Thomas' Hospital, London, United Kingdom

36 Hospital Virgen de la Arrixaca de Murcia, Inborn Metabolic Disease Unit, El Palmar, Spain

37 Manchester Academic Health Science Centre, University of Manchester, Willink Biochemical Genetics Unit, Genetic Medicine, Manchester, UK

38 Erasmus MC-Sophia Kinderziekenhuis, Erasmus Universiteit Rotterdam, Rotterdam, Netherlands 